\title{
Anestesia intravenosa en urgencia
}

\section{Total intravenous anesthesia in emergency}

Pablo O. Sepúlveda Voullieme'

\begin{abstract}
In emergency settings, TIVA should be adjusted in accordance to the pharmacological changes observed in the hypovolemic patient. With the understanding that in those cases there is a decrease in drug requirements, there is a tendency to underdose these patients, increasing the possibility of awareness. Variation in central volume concentration and blood flow redistribution increase plasma drug concentration (Cp). Decreased liver and kidney perfusion, hemodilution and hypothermia, all affect metabolism and clearance of drugs. Changes in drug bioavailability are also observed secondary to changes in plasma protein concentration and acidosis. Changes in pharmacodynamic of target organs are the product of metabolic and temperature disturbances. Due to the nature of hypovolemic shock, most systematic studies have been done in animals. In this condition, the volume of distribution and clearance of fentanyl decreases, increasing decremental times hence requiring bolus and infusion adjustment. Similar changes are observed when using remifentanil, but in this case its contextual half-life is not altered. In the case of etomidate use, most changes are observed on V2 and $\mathrm{V} 3$, with a minimal pharmacodynamic variation is observed, thus, requiring no adjustment. When propofol is used, the increase in $\mathrm{Cp}$ is proportional to the degree of hypovolemia, adding an increased sensitivity when it reaches over 40\%. Data fit to the Eleveld's model (from animal data extrapolation) and simulations in TIVA trainer are shown. Experience shows that these suggestions overestimate the dose, especially within the first 10 minutes. Therefore, it is recommended to reduce the target by $50 \%$ in the case of crystalloid-based resuscitation and by $20 \%$ when colloids are preferred. Finally, ketamine has been repositioned as an analgesic drug, and is not recommended as a hypnotic, except when used with propofol or benzodiazepines. For propofol, a staggered induction is recommended (together with remifentanil and a neuromuscular blocker), maintaining then the concentration at the site of effect with which the unconsciousness was achieved. The use of EEG monitoring will yield a better titration.
\end{abstract}

\section{RESUMEN}

En escenarios de urgencia, la TIVA se debe ajustar a los cambios farmacológicos observados en el paciente hipovolémico. En el entendido de que los requerimientos de drogas bajan, se tiende a subdosificar y con ello, aumentar la posibilidad de recuerdo intraoperatorio. La concentración del volumen central y la redistribución de flujo, aumentan la concentración plasmática $(C p)$ de droga. La disminución del flujo hepático y renal, la hemodilución y la hipotermia afectan el metabolismo y la distribución de las drogas. Cambios en la biodispo-

Hospital Base San José, Osorno.

Fecha de recepción: 21 de noviembre de 2020

Fecha de aceptación: 23 de noviembre de 2020

\section{ORCID}

0000-0003-2443-261X

Correspondencia:

Pablo O. Sepúlveda Voullieme

pasevou@gmail.com 
nibilidad de drogas también se observan secundario a cambios en la concentración de proteínas plasmáticas y acidosis. Cambios farmacodinámicos de los órganos blancos son producto de las alteraciones metabólicas y de temperatura. Por la naturaleza del shock hipovolémico, los estudios sistemáticos han sido fundamentalemente en animales. El volumen de distribución y aclaramiento del fentanilo disminuyen, aumentando los tiempos decrementales. Requiere ajuste de bolo e infusión. Los mismos cambios se observan en el remifentanilo, sin embargo, su vida media contextual no se altera. Para etomidato se observa una contracción centrada en V2 y V3 y una variación farmacodinámica mínima, por lo que no requiere ajuste. Con propofol el aumento de la $\mathrm{Cp}$ es proporcional a la hipovolemia, agregándose aumento de la sensibilidad cuando es mayor del $40 \%$. Se muestran ajustes basados en el modelo de Eleveld (de la extrapolación de datos animales) y simulaciones en TIVAtrainer. La experiencia muestra que estas sugerencias sobrestiman la dosis, especialmente los primeros 10 minutos, por lo que se aconseja disminuir el target en $50 \%$ en el caso de la reanimación basada en cristaloides y en $20 \%$ cuando se prefieren coloides. Por último, la ketamina se ha reposicionado como droga analgésica, no se recomienda como hipnótico, salvo en presencia de propofol o benzodiacepinas. Para propofol, se recomienda una inducción escalonada (junto con remifentanilo y un bloqueador neuromuscular), manteniendo luego con la concentración en en el sitio de efecto (Ce) con la que se alcanzó el LOC. El monitoreo con EEG permitirá una mejor titulación.

\section{L}

a anestesia intravenosa moderna ya cuenta con más de 20 años desarrollando modelos computarizados para la administración de fármacos para la inconsciencia y el control antinocioceptivo.

El uso de la anestesia intravenosa en cirugía no programada no se diferencia conceptualmente de la técnica usada en pacientes electivos. La diferencia radica en el ajuste a las condiciones fisiopatológicas que modifican la cinética y eventualmente la farmacodinamia.

El escenario de la urgencia absoluta o relativa hace sí tener más claridad de como se modifica dinámicamente en el transcurso de la cirugía la distribución y eliminación de drogas con la reanimación o el uso de drogas de apoyo hemodinámico. Estos conceptos no son diferentes en anestesia inhalada, aunque pueden tener particularidades en la vía de administración y captación del gas.

Debemos eso si destacar que hoy en día, a nuestro entender, no existe una verdadera anestesia inhalatoria pura. Ésta es a lo más una anestesia mayoritariamente intravenosa donde se administra un hipnótico inhalado y en concentraciones sólo requeridas para su efecto cortical. El uso de inhalados en concentraciones inmovilizantes es abiertamente excesivo para la depresión cortical y explica muchos de sus efectos de alteraciones cognitivas postoperatorias.

El conocer la farmacología de las drogas intravenosas es por lo tanto fundamental en el ambiente de urgencias.

Los modelos de Propofol iniciales incluidos en los TCI (iniciales de Target Controlled Infusion) eran sencillos, éstos eran modelos poblacionales de distribución y eliminación que calculaban la concentración plasmática en base a una única covariable como el peso[1], pero ésta resultó ser una simplificación excesiva, siendo útil solo para población adulta joven y mesomorfa. Se empezaron a desarrollar entonces modelos de mayor exigencia, como incluir edades extremas y obesidad[2],[3],[4].

Hoy estos modelos son adecuadamente predictivos, destacando el modelo construido con todos los aportes mundiales de datos, el llamado modelo de Eleveld[5].

La mayoría de las drogas intravenosas han ido sufriendo la misma evolución, aunque los datos en pacientes que requieren cirugías de urgencia son poco sistemáticos por las dificultades en desarrollar protocolos comparables y éticamente aceptables.

De todas maneras, estos patrones de corrección, que cuentan también con un modelo que describe una teórica concentración en sitio efector (relación Cp con un efecto determinado), no han podido superar tres problemas importantes:

a) La descripción inadecuada de la fase de inducción en condiciones de alta velocidad. Esto es así por la dificultad de describir la fase de mezcla adecuadamente. El resultado es que al inducir en forma rápida y en especial usando dianas referenciales $\left(E C_{50-95}\right)$, es frecuente sobredosificar el efecto. Esto es un especial desafío a superar en la inducción rápida del paciente con estómago lleno que será discutida más adelante.

b) La dificultad de contar con una señal biológica para describir el complejo fenómeno de la inconsciencia (desactivación de la capacidad perceptiva por pérdida de conectividad que genera integración de información compleja).

c) Los datos fueron obtenidos solo en pacientes electivos, lo que no representa al paciente hipo- 
volémico y/o sangrante. Éste será otro punto a desarrollar más adelante.

El propofol ha sido estudiado en condiciones de hipovolemia, pero en estudios en animales de forma controlada, al igual que las drogas analgésicas como los opioides. El conocer el sentido del cambio de los modelos de las perfusoras $\mathrm{TCl}$ es fundamental para realizar el ajuste en el paciente sangrante. Para eso se debe hacer una aproximación fisiopatológica inicial que destaca que la actitud para las drogas inhaladas e intravenosas son muy diferentes para entender el sentido de los cambios a realizar.

En 1978, Harrison presentó un estudio realizado durante 10 años donde muestra que la inducción anestésica era la primera causa de muerte en pacientes hipovolémicos[6]. Por otra parte, Weiskopf demostró que hemorragias de $30 \%$ de la volemia producían una disminución significativa de los requerimientos anestésicos: 40\% de ketamina y 33\% de tiopental[7]. En el caso del midazolam, un estudio en animales demostró que la administración de un bolo de $10 \mathrm{mg} \cdot \mathrm{kg}^{-1}$ en hipovolemias de $30 \%$ produce un aumento de la vida media y disminución del aclaramiento, sin cambios en el volumen de distribución $(\mathrm{Vd})$, pero potenciando el efecto hipotensor[8].

Uno de los problemas en los pacientes en shock hipovolémico es que se tiende a subdosificar las drogas infundidas, debido a su inestabilidad hemodinámica y al desconocimiento del ajuste de las drogas. Este hecho aumenta el riesgo de recuerdo intraoperatorio en esta población, que se elevaría a cifras de entre el $11 \%$ y el $43 \%$ [8]. En estos pacientes el uso de los nuevos EEG clínicos con espectrografía, sería fundamental para una buena titulación inicial y el ajuste dinámico durante la volemización.

Generalmente, la forma de administración de drogas intravenosas son los bolos de benzodiacepinas y opioides. En ocasiones se utiliza ketamina por su estabilidad hemodinámica y un bloqueador neuromuscular. En estos casos se evidenciarán los riesgos de sobredosis de ketamina y parálisis residual en el postoperatorio.

Mito aparte es que se considera a las benzodiacepinas mejores hipnóticos que propofol. Esto no es así. Estudios muestran que en condiciones normales el EC95 de propofol para amnesia de palabras es 0,9 $\mathrm{ug} \cdot \mathrm{ml}^{-1}$. Es probable que, en pacientes en estado de shock, basten $0,5 \mathrm{ug} \cdot \mathrm{ml}^{-1}$ para cubrir todo el espectro poblacional del hipovolémico agudo[10]. Debe tenerse presente que el tiempo de efecto máximo tras un bolo de midazolam es de 12 minutos, tiempo inadecuado para bloquear la experiencia y el recuerdo.

\section{Fisiopatología del shock hemorrágico}

Aunque el tema no es objeto de este artículo, es necesario enfatizar aquí que la pérdida de volumen intravascular gatilla una respuesta sistémica predecible mediada tanto por señales locales del lecho vascular periférico, como del sistema neuroendocrino, que se refleja en la farmacocinética de todas las drogas.

Los cambios fundamentales en la hemodinamia son: 1) depleción de volumen intravascular, lo que a su vez produce; 2 ) limitación del llene ventricular, el volumen de eyección, índice cardíaco y transporte de $\mathrm{O}_{2}$, todo lo que conduce finalmente a isquemia tisular; 3) activación de mecanismos compensatorios con vasoconstricción y estimulación simpática, estado hiperdinámico e hipermetabólico; 4) liberación de perpetuadores secundarios de isquemia tisular con agregación intravascular y microtrombosis y 5) liberación de perpetuadores terciarios de reperfusión con la formación de radicales superóxidos tóxicos

La pérdida de volumen circulante trae aparejada diversas alteraciones fisiopatológicas como son:

- Caída del débito cardiaco (DC) por caída de la precarga y posteriormente, del inotropismo producto de las alteraciones metabólicas. Esto tiene directo impacto en contraer el volumen central $\left(V_{1}\right)$, provocando un aumento de la concentración en plasma (Cp) de las drogas.

- Mecanismos compensatorios que producen una redistribución de flujo, con variaciones en los tamaños de los volúmenes de distribución impactando también en el aumento de la Cp.

- Disminución del flujo hepático-renal (tasa de extracción). Los principales órganos involucrados en el aclaramiento de drogas son el hígado y los riñones. En las diferentes etapas del shock, estos órganos ven comprometida en grado variable su perfusión, y por ende, sus funciones. Esta alteración del aclaramiento metabólico puede ser consecuencia del déficit de flujo y/o del cambio de fracción libre de drogas, afectando el acceso de las drogas a las enzimas y a la capacidad metabólica intrínseca del hígado. Las drogas de alta tasa de extracción se afectan por la caída del flujo, provocando un aumento de droga libre sin aumento total (el ejemplo es el mismo propofol). Las drogas de baja extracción (dependientes del aclaramiento intrínseco) se afectan transitoriamente por aumento de droga libre (diazepam, metadona). A lo anterior se agregan los efectos de la reanimación misma como la hemodilución y la hipotermia que afectan tanto el metabolismo como la distribución de las drogas (Tabla 1). 
- Disminución o aumento de distintas proteínas plasmáticas y cambios de fijación de drogas a ellas, lo que en algunos casos lleva a disponer de mayor droga libre o viceversa, con el consiguiente aumento o disminución de su efecto. Aumento de proteínas reactivas de fase aguda (alfa 1 glicoproteína ácida) disminuye droga libre básica como alfentanil. La disminución de albumina por hemodilución, aumenta droga libre ácida como propofol.

- Acidosis metabólica: disminuye la distribución de drogas ionizables (relajantes musculares).

- Finalmente, cambios farmacodinámicos de los órganos blancos de las drogas anestésicas, producto de las alteraciones metabólicas y de temperatura.

\section{Cambios farmacocinéticos específicos por drogas}

Lo crítico e inesperado de la situación que se vive en las hipovolemias agudas limita realizar estudios clínicos estandarizados en humanos. Eso ha llevado a realizar ésto en animales con intentos de extrapolar sus resultados, pero existen complejidades agregadas en los modelos estudiados: los animales ya se encuentran anestesiados al iniciar los estudios, pudiéndose generar sesgos en la interpretación del comportamiento farmacológico y las correcciones de los modelos a poblaciones humanas se hacen en base a otras covariables y datos del EEG.

Antes de hacer referencia a la anestesia intravenosa, es necesario discutir brevemente algo sobre las drogas inhaladas, que tienen cambios muy diferentes a las intravenosas. Es importante considerar esto dado que generalmente son de sentido inverso.

\section{Drogas inhaladas}

Los cambios en la cinética de los agentes anestésicos inhalatorios en hipovolemia aguda presentan algunas particularidades. El aumento del shunt pulmonar por hipovolemia junto con la caída de débito cardíaco, producen una disminución de la captación de gases, y un acortamiento del tiempo de equilibrio $\mathrm{Fe} / \mathrm{Fi}$, generando una falsa sensación de equilibrio sanguíneo del gas y de tener mayor efecto anestésico. La fase de reanimación provoca un efecto inverso de reperfusión y una captación aumentada de gas, generando en estos pacientes mayor presión parcial en el plasma y sobredosificación.

Los estudios con isoflurano en hipovolemias de $40 \%$ de pérdida sanguínea demuestran un significativo aumento de la potencia de la droga. El EC ${ }_{50}$ de isoflurano con frecuencia espectral disminuyó de 1,12\% a 0,99\%, aún después de la reanimación con coloides. Junto a lo anterior, los tiempos de equilibrio de la Fe/ Fi disminuyeron producto de la menor captación de gas, producto de la disminución del débito cardíaco, dando una falsa impresión de mayor profundidad anestésica[11],[12].

El estudio más reciente en un modelo en cerdos sangrados y anestesia con isoflurano muestra que con hipo débito y lactacidosis se produce una disminución de la CAM poblacional, aunque sin marcada hipotensión, y la reanimación con coloides recupera la hemodinamia, pero no corrige la CAM[13].

Por ahora es complejo comparar las diferencias entre los efectos farmacodinámicos de los hipnóticos intravenosos y agentes inhalatorios en esta condición clínica. Los datos son escasos y además las drogas inhaladas han sido evaluadas solo hasta una hipovole-

\begin{tabular}{lll}
\multicolumn{2}{c}{ Tabla 1. Tasa de extracción hepática de diferentes fármacos usados en anestesia, clasificados por su grado de } \\
eliminación
\end{tabular}

De Paepe P. Belpaire FM, Van Hoey G. et al. J Pharmacol Exp Ther 1999; 290:1048-53. 
mia no mayor al $40 \%$, a diferencia del propofol, donde se ha llegado a $65 \%$ de sangrado. Sin embargo, se ha observado algunas diferencias particulares, por ejemplo, el efecto inmovilizante espinal de las drogas inhaladas se potencia más que por propofol en el shock hipovolémico[14].

Por otra parte, la metodología de los estudios ha sido diferente. El tipo de procesamiento EEG usado es distinto. El isoflurano fue evaluado con SEF (del inglés, Spectral Edge Frequency) en cerdos y se alcanzaron depresiones mayores a $20 \mathrm{~Hz}$. En anestesia en humanos se exigen valores alrededor de 10-12 $\mathrm{Hz}$ (estado alfa con propofol), y fue evaluado con BIS (del inglés, Bispectral Index), llegándose incluso a la supresión eléctrica. Otra diferencia de metodología ha sido la forma de evaluar la reanimación y su impacto en los cambios farmacodinámicos posteriores. Los estudios de shock hipovolémico que incluyen análisis farmacodinámico con isoflurano fueron reanimados con coloides, en cambio en el caso del propofol solo hay datos farmacodinámicos de reanimación con ringer lactato 1:1.[15].

\section{Drogas intravenosas}

\section{Fentanilo}

Una de las primeras drogas estudiadas para un shock isobárico (se mantiene en hipotensión estable) fue el fentanilo[16]. Cerdos de $70 \mathrm{~kg}$ fueron sangrados cerca de 2.000 cc (40\% de la volemia hasta alcanzar una PAM de $40 \mathrm{mmHg}$ durante una hora), a los que se les administró fentanilo en una perfusión durante $5 \mathrm{~min}$. Los cambios farmacocinéticos más destacados fueron la disminución del aclaramiento metabólico hepático y la contracción de los Vd central y $V_{2}$ (Tabla 2). Estas reducciones explican, al menos parcialmente, que la $\mathrm{Cp}$ se duplicara a los $5 \mathrm{~min}$ de perfusión, llevando a una disminución del $50 \%$ del requerimiento de fentanilo con relación al grupo control no sangrado.

Otro cambio muy notorio es la prolongación en los tiempos decrementales del fentanilo, recalculados en base a la cinética del estado hipovolémico agudo. Así, el decaimiento al $50 \%$ en los cerdos control fue de 60 min tras 200 min de infusión, en tanto que en los cerdos sangrados se prolongó a 100 min.

En términos prácticos, en una simulación (Figura 1), manteniendo las proporciones de los cambios y usando el modelo de adultos de Shafer, implicaría que debe reducirse la dosis de bolos de fentanilo a casi la mitad y las de infusión en 30\%.

\section{Remifentanilo}

Para estudiar remifentanilo, se sangraron 16 cerdos a una presión arterial media de $40 \mathrm{mmHg}$ logrando una hipovolemia del $65 \%$. Luego se procedió a infundir $10 \mathrm{ug} \cdot \mathrm{kg}^{-1} \cdot \mathrm{min}^{-1}$ por $10 \mathrm{~min}[7]$.

En este caso también se observó una contracción del Vd central y una caída en el aclaramiento, pero sin prolongación mayor en la vida media contextual. Desde el punto de vista práctico, se concluye que debiera reducirse el aporte de remifentanilo al menos $40 \%$ para alcanzar igual $\mathrm{Cp}$.

Este estudio incluyó una evaluación farmacodinámica con SEF que mostró que no habría cambios en la farmacodinamia de remifentanilo.

\section{Etomidato}

El etomidato es reconocido como de alta estabilidad hemodinámica en la inducción, pero no se recomienda su uso en perfusión. Existen dos estudios que han evaluado el comportamiento farmacológico del etomidato en hipovolemia aguda. En cerdos sangrados hasta $42 \%$ de la volemia para alcanzar una presión arterial media de $50 \mathrm{mmHg}$, se infundió por $10 \mathrm{~min} 300 \mathrm{ug} \cdot \mathrm{kg}^{-1} \cdot \mathrm{min}^{-1}$ de etomidato y se muestreó la $\mathrm{Cp}$ arterial. Se demostró que el cambio farmacocinético es proporcional al volumen de sangrado, con una contracción centrada en $V_{2}$ y $V_{3}$ y con una variación farmacodinámica que sería mínima, por lo que no sería necesario en este caso el ajuste del bolo[18].

\section{Propofol}

Kazama[19] infundió $2 \mathrm{mg} \cdot \mathrm{kg}^{-1} \mathrm{~h}^{-1}$ de propofol en cerdos de $30 \mathrm{~kg}$ y luego los sangró progresivamente hasta extraer cerca del $50 \%$ de la volemia. Los animales presentaron un comportamiento hemodinámico bifásico. Tanto la resistencia vascular sistémica como

\begin{tabular}{|c|c|c|c|c|c|c|}
\hline & $v_{1}$ & $v_{2}$ & $v_{3}$ & $\mathrm{Cl}_{1}$ & $\mathrm{Cl}_{2}$ & $\mathrm{Cl}_{3}$ \\
\hline Control & 12,6 & 16,8 & 162 & 1,63 & 2,56 & 2,09 \\
\hline Shock & 5,6 & 6,35 & 160 & 0,65 & 1,14 & 1,64 \\
\hline
\end{tabular}




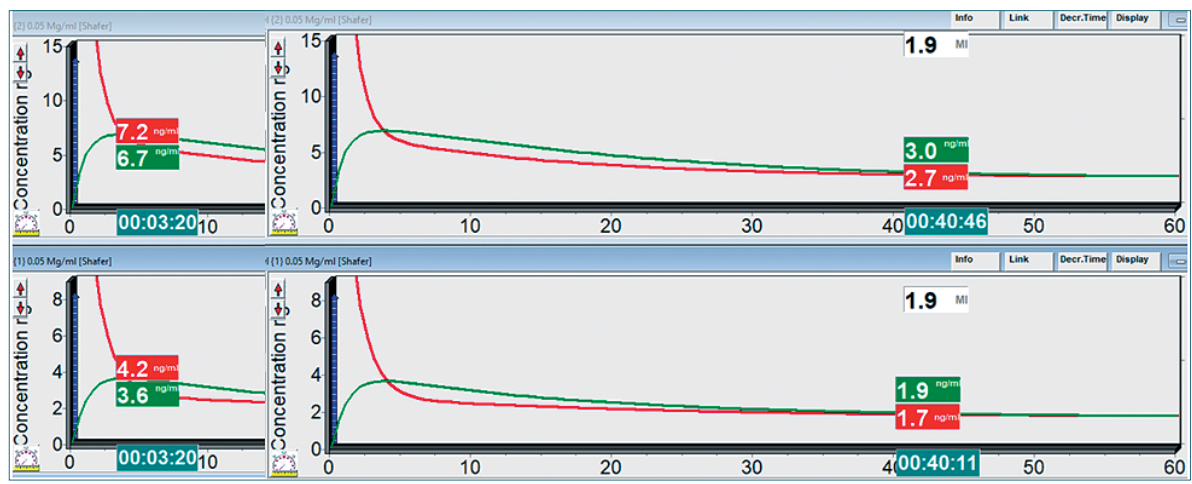

Figura 1. Bolo de $3 \mathrm{ug} \cdot \mathrm{kg}^{-1}$ de fentanilo, seguido de una perfusión de $2 \mathrm{ug} \cdot \mathrm{kg}-1 \cdot \mathrm{h}^{-1}$ (arriba paciente en shock $40 \%$ y abajo paciente control).

la frecuencia cardíaca aumentaron hasta un punto de colapso cuando se alcanzó alrededor del $50 \%$ de la volemia. El débito cardiaco cayó progresivamente a medida que se hipovolemizaba. Mientras los cerdos iban siendo sangrados la Cp de propofol aumentó progresivamente, de modo que:

- Con un sangrado hasta $25 \%$, no hubo variación de Cp.

- Si el débito cayó 31\%, y se extrajo entre el 25 y el $30 \%$ de la volemia, la Cp aumentó $20 \%$.

- Si el débito cayó $46 \%$, y se extrajo entre el 30 y el $40 \%$ de la volemia, la Cp aumentó $46 \%$.

- Al entrar en fase de descompensación con una hemorragia mayor al 50\%, la Cp aumentó 3,75 veces.

En estudios realizados con BIS los animales expuestos a hipovolemia mayor al 40\%, muestran un gran aumento de la sensibilidad a propofol[20].

La reanimación sí tiene impacto farmacológico según la forma en que es realizada. Por ejemplo, si después de sangrar a los cerdos un $60 \%$ de la volemia y/o llegar a una presión arterial media de $40 \mathrm{mmHg}$, se realizó una reposición de volumen con coloides 1:1 o cristaloides 3:1 y se observó que la $\mathrm{Cp}$ de propofol no es distinta a la del grupo control gracias a que se mantenía un débito cardíaco normal. Pero en el grupo reanimado exclusivamente con cristaloides 1:1 el débito se derrumbó progresivamente, colapsando los animales mucho antes que el grupo reanimado con coloides[21]. Se concluyó que la $\mathrm{Cp}$ es dependiente de cómo y con qué se realiza la reanimación. Lamentablemente, en este trabajo no se estudió la alteración farmacodinámica posterior a la reanimación con coloides.

El aumento de droga libre, producto de la hipoalbuminemia y alteraciones metabólicas puede ser responsable en parte del aumento del efecto del propofol.
Un reporte de cuatro casos de hemorragia no esperada durante trasplante hepático, observó que el aumento de la $\mathrm{Cp}$ de propofol total era de 2 veces, pero el aumento de droga libre llegaba a ser 4 veces mayor[22]. Esto podría explicar que la alteración farmacodinámica evaluada era en realidad mayormente por este cambio farmacocinético. Otros estudios en circulación extracorpórea muestran efectos similares disminuyendo la albúmina y aumentando la droga libre[23].

Dado que se suma desde ahora un nuevo modelo de propofol que incorpora todas las covariables de estudios previos, el modelo Eleveld, se hará los cálculos de ajuste para poblaciones en base a este modelo. Usando la parametrización que usa Johnson en cerdos sangrados $40 \%$ de la volemia[20] observaríamos los siguientes cambios. Este ajuste es una aproximación orientativa solamente (Tabla 3).

Las Figuras 2A, 2B, 3A y 3B son simulaciones con el software Tivatrainer (www.eurosiva.com) para los cambios farmacocinéticos propuestos por los estudios y aplicados a humanos en forma teórica como elemento referencial del impacto de la hipovolemia aguda. Se utiliza el modelo Eleveld que representa hoy el mejor modelo que integra covariables múltiples de edades y tamaños.

Nuestra experiencia personal, es que estas propuestas, si bien útiles, están significativamente sobre predichas, sobre todo al inicio de las perfusiones del $\mathrm{TCl}$. Es decir, las Cp son en realidad más bajas en los primeros $10 \mathrm{~min}$. Eso puede hacer pensar que el paciente requiere más droga y terminar sobredosificado. Es probable que la corrección clínica sea simplemente esperar más tiempo, en especial si estamos en un escenario donde no se necesita intubar en forma inmediata.

Como no es posible realizar ajustes de modelos en las perfusoras y estos datos son sólo aproximados, es 
posible guiarse por la tabla expuesta o simplemente reducir el target $50 \%$ para niveles de sangrado como los señalados.

La fórmula de corrección presentada es para las reanimaciones basadas en cristaloides. Si se decide el uso de coloides como prioritarios, la corrección de las masas totales y de target debería adecuarse a una reducción de alrededor del 20\% solamente.

\section{Ketamina}

La ketamina es una droga que ha sido ampliamente usada en hipovolemias agudas. Se ha usado como parte de las drogas en inducciones rápidas o estados asmáticos, en procedimientos traumatológicos breves mezclado con propofol, y en condiciones de conflicto bélico, o en los quemados, pero es útil recordar que pacientes con estados de depleción adrenérgica como shock séptico, la ketamina no es recomendada porque su ventaja de estabilidad hemodinámica está dada por la liberación de catecolaminas. En estos casos se evidencia su efecto depresor cardiovascular[24],[25].

Hoy en día la ketamina se ha reposicionado como una droga analgésica, y sus dosis se deben ajustar a su efecto mayoritariamente espinal y no cortical, para evitar los efectos secundarios postoperatorios (delirios, despersonalización, sueños desagradables). La ketamina no está indicada como hipnótico y requiere en ese caso ser complementada con propofol o una benzodiacepina, complicando aún más este escenario por esencia inestable.

La dosis analgésica recomendada en pacientes normovolémicos es de $0,3 \mathrm{mg} \cdot \mathrm{kg}^{-1} \cdot \mathrm{h}^{-1}$, que debiera

\begin{tabular}{|c|c|c|c|c|c|c|c|c|}
\hline $\begin{array}{l}\text { Tamaño } \\
\text { del ajuste }\end{array}$ & $\begin{array}{l}\text { Adulto } 45 \\
\text { años } 160 \\
\mathrm{~cm} 70 \mathrm{~kg}\end{array}$ & Ajuste & $\begin{array}{c}\text { Niño } 6 \\
\text { años } \\
110 \mathrm{~cm} 18 \\
\text { kg }\end{array}$ & Ajuste & $\begin{array}{c}\text { Anciano } \\
70 \text { años } \\
160 \mathrm{~cm} 70 \\
\text { kg }\end{array}$ & Ajuste & $\begin{array}{c}\text { Obeso } 45 \\
\text { años } \\
160 \mathrm{~cm} \\
100 \mathrm{~kg}\end{array}$ & Ajuste \\
\hline$V_{1}<22 \%$ & 9,5 & 7,41 & 5,2 & 4,05 & 8,6 & 6,7 & 9,5 & 5,2 \\
\hline V2 < 60\% & 27,7 & 11 & 8,6 & 3,44 & 21 & 8,4 & 37 & 14,8 \\
\hline V3 < 30\% & 134 & 93 & 38 & 26,6 & 134 & 93,8 & 153 & 107 \\
\hline $\mathrm{Cl} 1<50 \%$ & 90 & 45 & 37 & 18,5 & 90 & 45 & 115 & 57,5 \\
\hline $\mathrm{Cl} 2<78 \%$ & 79 & 17,4 & 56 & 12,32 & 53 & 11,6 & 97 & 21,3 \\
\hline $\mathrm{Cl} 3<50 \%$ & 35 & 17 & 17 & 8,5 & 29 & 14,5 & 37 & 18,5 \\
\hline
\end{tabular}

Vd en I y clearance en $\mid \bullet h^{1}$

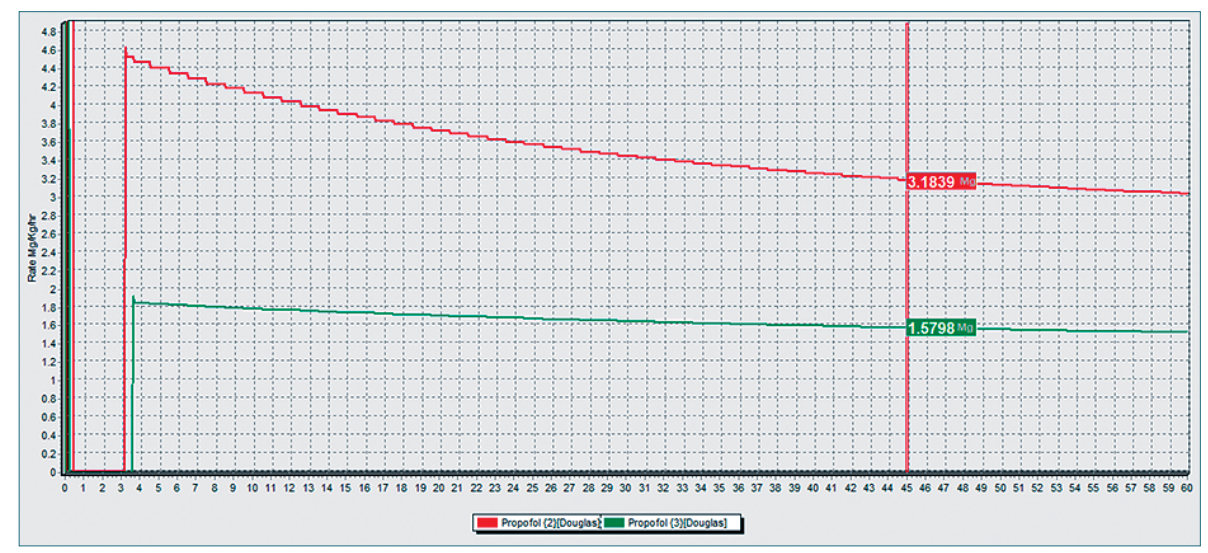

Figura 2A. Paciente de 45 años, obeso: en rojo normovolémico, en verde hipovolémico. Se muestra arriba masa acumulada para target de $2 \mathrm{ug} \cdot \mathrm{ml}^{-1}$ en el tiempo $\left(\mathrm{mg} \cdot \mathrm{kg}^{-1} \cdot \mathrm{h}^{-1}\right)$. También se indica la masa en $\mathrm{mg}$ totales a los $45 \mathrm{~min}$. 


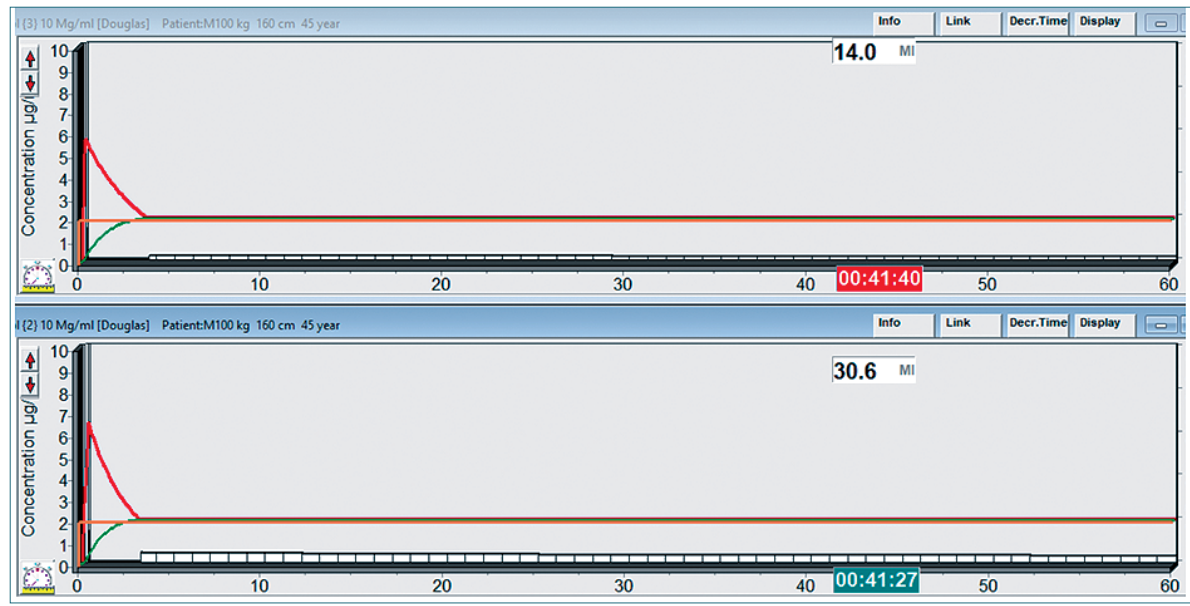

Figura 2B. Paciente obeso (arriba hipovolémico, abajo normovolémico). Masa de droga acumulada para target de $2 \mathrm{ug} \cdot \mathrm{ml}^{-1}$ a los $45 \mathrm{~min}$.

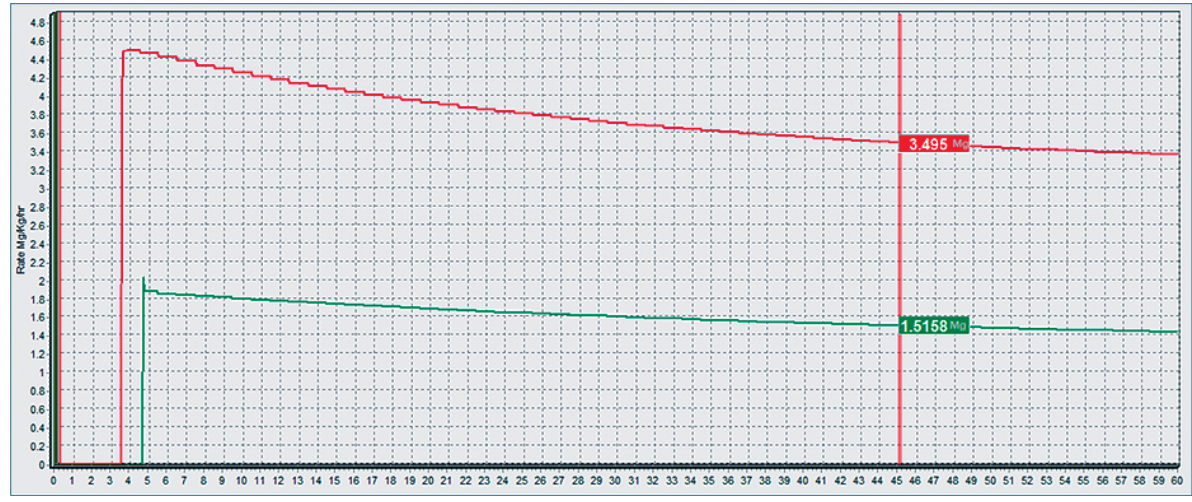

Figura 3A. Paciente anciano, en rojo normovolémico, en verde hipovolémico para mantener target de $2 \mathrm{ug} \cdot \mathrm{ml}^{-1}$.

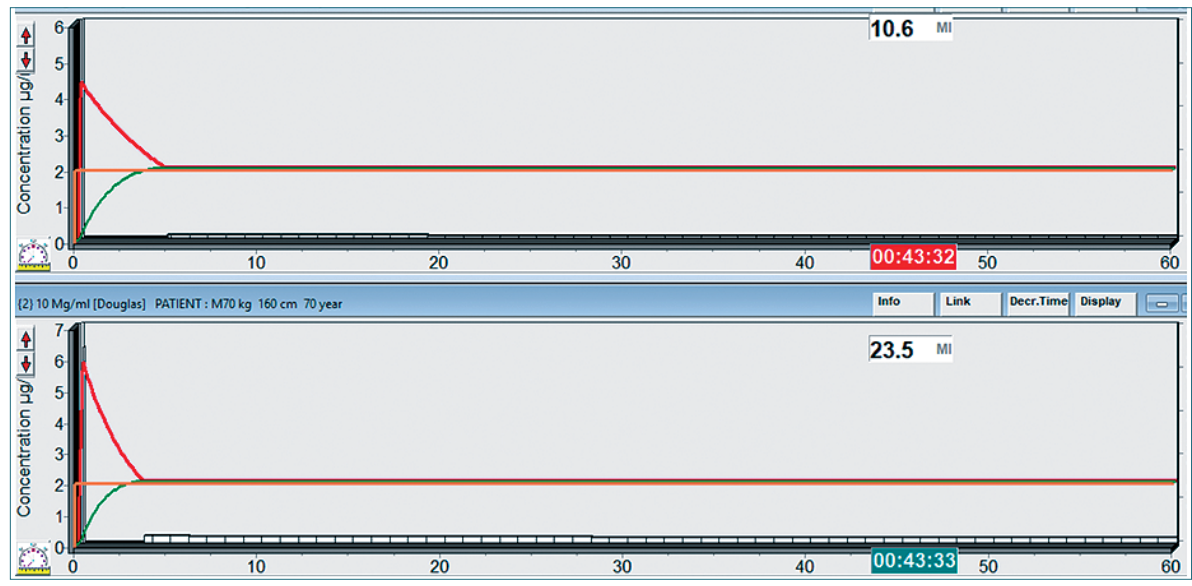

Figura 3B. Paciente anciano (arriba hipovolémico, abajo normovolémico) masa de droga acumulada a los $45 \mathrm{~min}$ para un target de $2 \mathrm{ug} \cdot \mathrm{ml}^{-1}$. 
ser ajustada a la condición de hipovolemia[7].

Vale la pena recordar que en la actividad cortical desencadenada por la ketamina predomina la actividad alta (beta 14 a $20 \mathrm{~Hz}$ ), lo que provoca una sensación de paciente superficial, tendiendo a aumentar el hipnótico (propofol o droga inhalada) en forma innecesaria.

\section{Algunas consideraciones sobre inducción rápida y TCl}

La primera recomendación que podemos hacer es el uso de etomidato, que en el escenario hipovolémico asociado a estómago lleno es una buena y muy estable droga. Tras la inducción podemos pasar a usar el $\mathrm{TCl}$ de propofol para el mantenimiento en las dianas recomendadas, y ojalá disponiendo de un EEG con espectrografía para la titulación.

Respecto al uso de $\mathrm{TCl}$ en la inducción, no existen estudios formales relacionados con esta materia, por lo que esta discusión se basa en la experiencia personal.

Tradicionalmente, la inducción rápida para intubar al paciente con estómago lleno se ha realizado con un bolo manual de un agente inductor (tiopental, propofol, etomidato o ketamina), un relajante muscular de tiempo de inicio rápido (succinilcolina o rocuronio en dosis tiempo equivalentes) y un opioide.

Esta técnica aparentemente sería más rápida porque supera la limitante de la perfusora (velocidad máxima de $1.200 \mathrm{ml} \cdot \mathrm{h}^{-1}$ ) permitiendo tener al paciente rápidamente inconsciente, relajado, con la vía aérea aislada en el menor tiempo posible para tener el menor riesgo de exposición a una regurgitación y aspiración de contenido gástrico.

La desventaja de esta aproximación es que, al usar un bolo manual, no podemos utilizar el TCl, porque este bolo altera el cálculo de las Cp. Además, otro problema secundario es la imposibilidad de titular el requerimiento específico del paciente. Esto es de especial importancia en casos complejos de pacientes ancianos con alta fragilidad de alteraciones cognitivas secundarias a una sobredosis.

La inducción alternativa que se propone usando $\mathrm{TCl}$, es realizar una inducción escalonada con propofol hasta un grado de sedación Ramsay 2, asegurándose que el paciente sea capaz de proteger su vía aérea con una prueba de deglución y tos antes del escalón que lo lleve a la pérdida de consciencia.

Las dianas habituales de este nivel de sedación están en alrededor de 1,5 a 1,8 ug. $\mathrm{ml}^{-1}$, situación en que el paciente está aún bien conectado al medio.
Desde esa condición, un bolo que aporte un target de 3,0 a 3,3 ug. $\mathrm{ml}^{-1}$, en combinación con remifentanil y el bloqueador neuromuscular es adecuado para alcanzar la inconsciencia (Figura 4). En la experiencia personal, los tiempos medidos para obtener condiciones de intubación adecuada comparando ambas técnicas, favorecen levemente a la técnica $\mathrm{TCl}$.

Las inducciones rápidas a target alto han mostrado requerir Ce calculadas cercanas a 4,5 ug. $\mathrm{ml}^{-1}$ en los modelos, con bolos de casi $2 \mathrm{mg} \cdot \mathrm{kg}^{-1}$. Los pacientes inducidos con cargas de bolos clásicos de $2 \mathrm{mg} \cdot \mathrm{kg}^{-1}$ requieren esa cantidad de droga por iniciar la carga desde cero y superar una fase de mezcla inestable. En inducciones escalonadas o técnica de infusión a tasa fija y luego $\mathrm{TCl}$ tras la inconsciencia (LOC), las Cp usadas rara vez sobrepasan de $3 \mathrm{ug} \cdot \mathrm{ml}^{-1}$.

Evaluaciones electroencefalográficas muestran estas masas de droga como un exceso, evaluado en el grado de depresión de las bandas alfa frontales del EEG. La inducción propuesta en este caso con esta sedación previa requiere de un salto mucho menor, no superior a alcanzar $3 \mathrm{ug} \cdot \mathrm{ml}^{-1}$, reduciendo la sobredosificación, sobrepasando en parte la fase de mezcla y mucho más cercana a las dianas de uso habitual[25]. En la Figura 5, se observa claramente un aumento en el poder de alfa LOC durante la inducción lenta de propofol. En contraste, la aparición del poder alfa ocurre unos minutos después de la LOC durante la infusión rápida de propofol, con gran depresión y poca estabilidad de actividad alfa.

Tras la pérdida de conciencia, la secuencia de relajante muscular y remifentanil en un target de 5 $\mathrm{ng} \cdot \mathrm{ml}^{-1}$ es la habitualmente usada. Para esta inducción, se recomienda flexibilidad y tacto clínico dado la variabilidad poblacional.

El escenario combinado de paciente hipovolémico y estómago lleno hace más importante realizar una mínima aproximación de titulación. Es probable que estos pacientes con Cp muy bajas de propofol ya pierdan la conciencia, en especial ancianos hipovolémicos. Existen, además, factores distorsionadores de los datos cinéticos. Por ejemplo, en pacientes con shock hipovolémico moderado y compensado, la hiperdinamia producto de la respuesta simpática, puede hacer que la inducción sea más lenta, al generar retraso en la mezcla de la droga (aumenta el tiempo de equilibrio $\mathrm{Cp}$-Cefecto descrita por los modelos), dando una impresión de mayor demanda. En realidad, las Cp alcanzadas serán más altas al estabilizarse la mezcla tras 10 o 15 min y el paciente estará sobredosificado y manifestará más alteraciones hemodinámicas asociadas.

La recomendación en estos pacientes hoy en día 


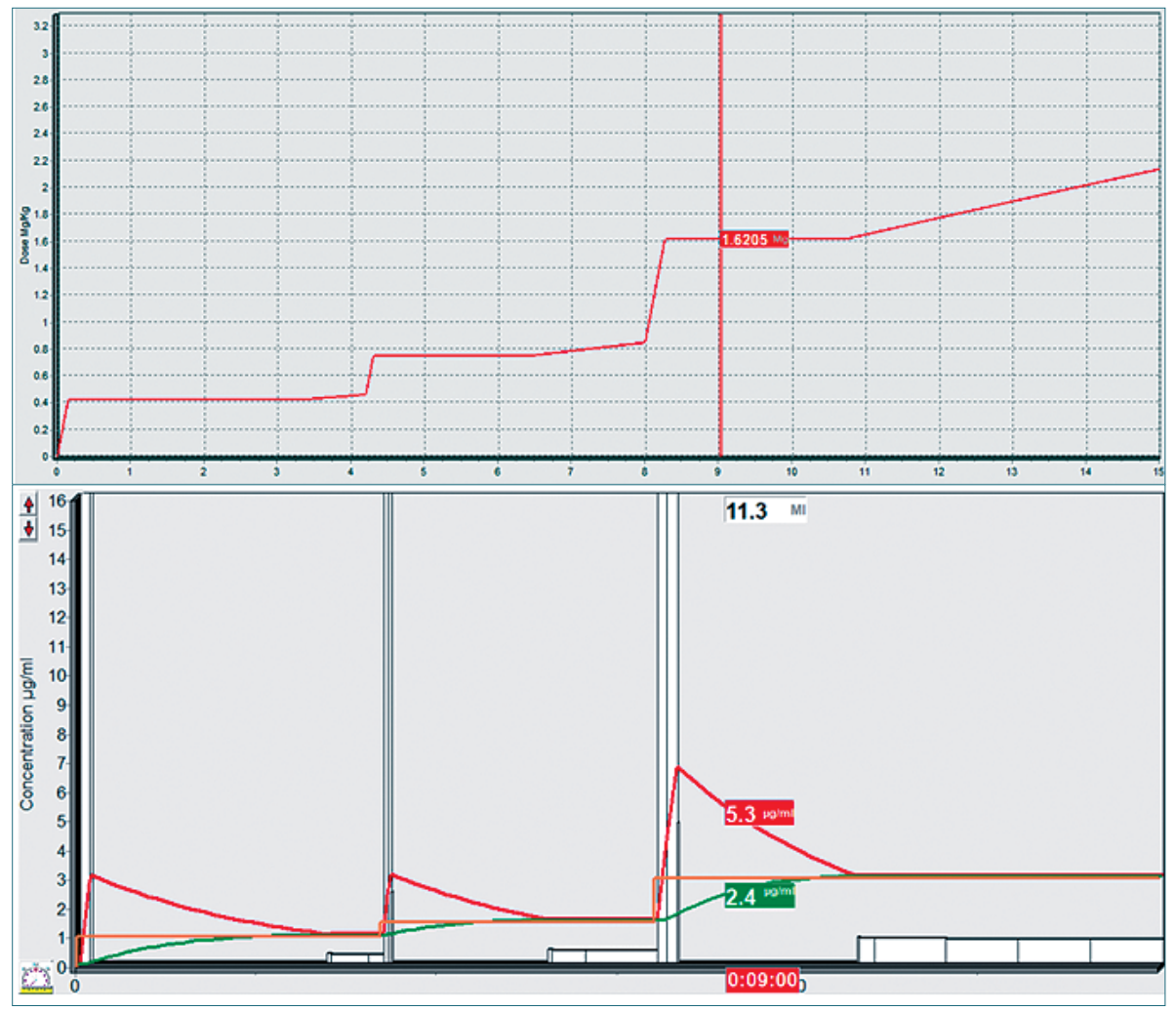

Figura 4. Gráficos paralelos: arriba, se observa consumo de propofol $\left(\mathrm{mg} \cdot \mathrm{kg}^{-1}\right)$ en el tiempo en una presedación de dos escalones, el tercer escalón es LOC. Se consume una masa total de $1,6 \mathrm{mg} \cdot \mathrm{kg}^{-1}$. Abajo, se observa la misma simulación usando $\mathrm{Cp} /$ Cefecto, donde se observa Ce de $2,4 \mathrm{ug} \cdot \mathrm{ml}^{-1}$ al LOC.

es agregar monitoreo EEG para realizar un ajuste más fino, que asuma la dinámica de la reanimación y el impacto sobre la cinética de las drogas. 

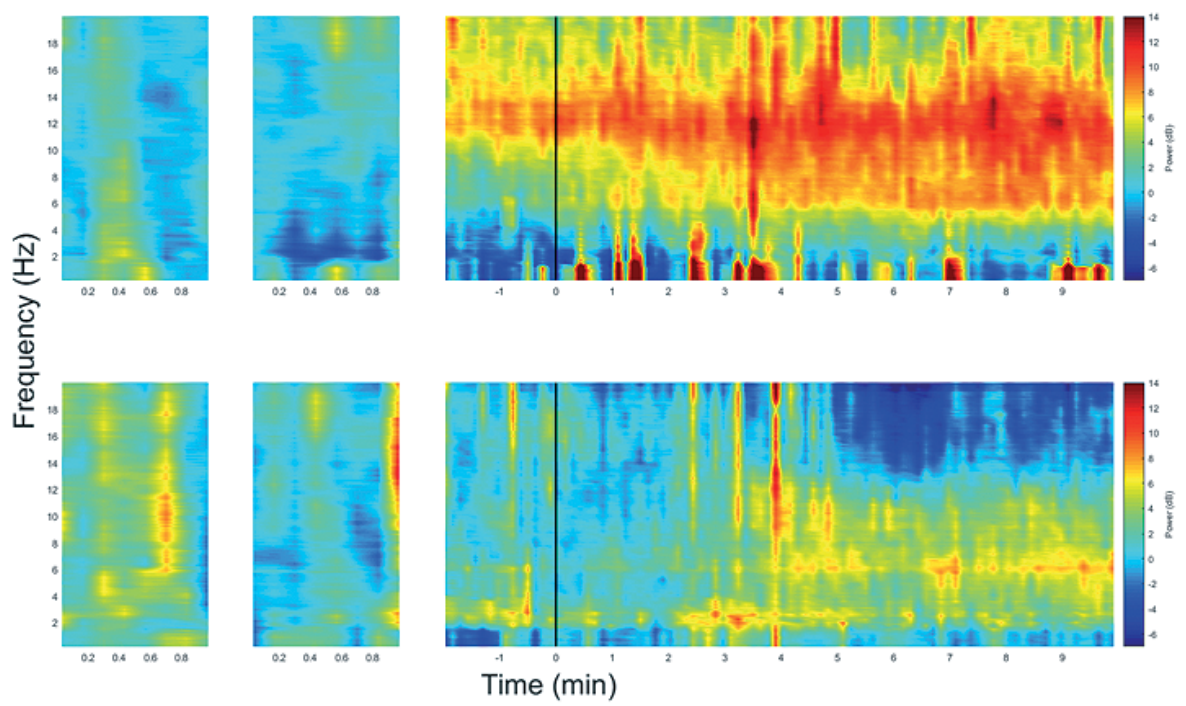

Figura 5. Gráfico de comportamiento de las frecuencias de actividad frontal, basal y tiempo 0 . Arriba: frecuencia de tiempo para la condición de perfusión lenta $10 \mathrm{mg} \cdot \mathrm{kg}-1 \cdot \mathrm{h}^{-1}$ hasta la pérdida de la conciencia (LOC) y manteniendo luego la Ce de LOC. Abajo: comportamiento de la actividad frontal en el tiempo tras una inducción rápida a 5,4 ug $\cdot \mathrm{ml}-1$. Se observa comportamiento de banda alfa muy diferente tras la administración de propofol al LOC (línea negra vertical) y hasta 10 min después. La inducción lenta muestra una banda alfa (rojo) muy intensa, que representa una adecuada condición de inconsciencia. La inducción rápida muestra un alfa tardío y luego débil evidenciando una fase de mezcla inestable con efecto no precisable, y una instalación posterior débil, probablemente por efecto de sobredosis de la alimentación troncal sobre el tálamo.

\section{Referencias}

1. Marsh B, White M, Morton N, Kenny GN. Pharmacokinetic model driven infusion of propofol in children. Br J Anaesth. 1991 Jul;67(1):41-8. https:// doi.org/10.1093/bja/67.1.41 PMID:1859758

2. Schnider TW, Minto CF, Gambus $\mathrm{PL}$, Andresen C, Goodale DB, Shafer SL, et al. The influence of method of administration and covariates on the pharmacokinetics of propofol in adult volunteers. Anesthesiology. 1998 May;88(5):1170-82. https:// doi.org/10.1097/00000542199805000-00006 PMID:9605675

3. Cortínez LI, Sepúlveda P, Rolle A, Cottin P, Guerrini A, Anderson BJ. Effect-Site Target-Controlled Infusion in the Obese: Model Derivation and
Performance Assessment. Anesth Analg. 2018 Oct;127(4):865-

72. https://doi.org/10.1213/

ANE.0000000000002814

PMID:29401079

4. Absalom A, Kenny G. 'Paedfusor' pharmacokinetic data set. $\mathrm{Br}$ J Anaesth. 2005 Jul;95(1):1103. https://doi.org/10.1093/bja/ aei567 PMID:15941735

5. Eleveld DJ, Colin P, Absalom AR, Struys MM. Pharmacokinetic-pharmacodynamic model for propofol for broad application in anaesthesia and sedation. Br J Anaesth. 2018 May;120(5):942-59. https://doi. org/10.1016/j.bja.2018.01.018 PMID:29661412

6. Harrison GG. Death attributable to anaesthesia. A 10-year survey (1967-1976). Br J Anaesth. 1978 Oct;50(10):1041-6. https://doi. org/10.1093/bja/50.10.1041 PMID:708545
7. Weiskopf RB, Bogetz MS. Haemorrhage decreases the anaesthesic requirements for ketamine and thiopentone in the pig. $\mathrm{B} J$ Anaesthesia 1985; 57: 1022-5.

8. Adams P, Gelman $S$, Reves JG, Greenblatt DJ, Alvis JM, Bradley E. Midazolam pharmacodynamics and pharmacokinetics during acute hypovolemia. Anesthesiology. 1985 Aug;63(2):140-6. https:// doi.org/10.1097/00000542198508000-00004 PMID:3161424

9. Bogetz MS, Katz JA. Recall of surgery for major trauma. Anesthesiology. 1984 Jul;61(1):6-9. https://doi. org/10.1097/00000542198461010-00003 PMID:6742485

10. Veselis RA, Reinsel RA, Feshchenko VA, Wronski M. The comparative amnestic effects of 
midazolam, propofol, thiopental, and fentanyl at equisedative concentrations. Anesthesiology. 1997 Oct;87(4):749-64. https:// doi.org/10.1097/00000542199710000-00007 PMID:9357875

11. Kurita T, Morita K, Fukuda K, Uraoka M, Takata K, Sanjo Y, et al. Influence of hypovolemia on the electroencephalographic effect of isoflurane in a swine model. Anesthesiology. 2005 May;102(5):948-53. https:// doi.org/10.1097/00000542200505000-00013 PMID:15851881

12. Kurita T, Morita K, Fukuda K, Uraoka M, Takata K, Sanjo $Y$, et al. Influence of hemorrhagic shock and subsequent fluid resuscitation on the electroencephalographic effect of isoflurane in a swine model. Anesthesiology. 2005 Dec;103(6):1189-94. https:// doi.org/10.1097/00000542200512000-00013 PMID:16306731

13. Kurita T, Takata K, Uraoka M, Morita K, Sanjo Y, Katoh T, et al. The influence of hemorrhagic shock on the minimum alveolar anesthetic concentration of isoflurane in a swine model. Anesth Analg. 2007 Dec;105(6):163943. https://doi.org/10.1213/01. ane.0000287252.39383.17 PMID:18042861

14. Kurita T, Takata K, Morita K, Morishima Y, Uraoka M, Katoh $T$, et al. The influence of hemorrhagic shock on the electroencephalographic and immobilizing effects of propofol in a swine model. Anesth Analg. 2009 Aug;109(2):398404. https://doi.org/10.1213/ ane.0b013e3181a96f9a PMID:19608809

15. Johnson KB, Egan TD, Kern SE, McJames SW, Cluff ML, Pace NL. Influence of hemorrhagic shock followed by crystalloid resuscitation on propofol: a pharmacokinetic and pharmacodynamic analysis. Anesthesiology. 2004 Sep;101(3):647-59. https:// doi.org/10.1097/00000542200409000-00013 PMID:15329589

16. Egan TD, Kuramkote S, Gong G, Zhang J, McJames SW, Bailey PL. Fentanyl pharmacokinetics in hemorrhagic shock: a porcine model. Anesthesiology. 1999 Jul;91(1):156-66. https:// doi.org/10.1097/00000542199907000-00024 PMID:10422941

17. Johnson KB, Kern SE, Hamber EA, McJames SW, Kohnstamm KM, Egan TD. Influence of hemorrhagic shock on remifentanil: a pharmacokinetic and pharmacodynamic analysis. Anesthesiology. 2001 Feb;94(2):322-32. https:// doi.org/10.1097/00000542200102000-00023 PMID:11176098

18. Johnson KB, Egan TD, Layman J, Kern SE, White JL, McJames SW. The influence of hemorrhagic shock on etomidate: a pharmacokinetic and pharmacodynamic analysis. Anesth Analg. 2003 May;96(5):1360-8. https://doi.org/10.1213/01. ANE.0000055804.30509.69 PMID:12707134

19. Kazama T, Kurita T, Morita K et al. Influence of hemorrhage on propofol pseudo-steady state concentration. Anesthesiology 2002; 97: 1156:61 https:// doi.org/10.1097/00000542200211000-00020.

20. Johnson KB, Egan TD, Kern SE, White JL, McJames SW, Syroid N, et al. The influence of hemorrhagic shock on propofol: a pharmacokinetic and pharmacodynamic analysis. Anesthesiology. 2003 Aug;99(2):409-20. https:// doi.org/10.1097/00000542-

\section{0-00023}

PMID:12883414

21. Kurita T, Kazama T, Morita K, Fujii S, Uraoka M, Takata K, et al. Influence of fluid infusion associated with high-volume blood loss on plasma propofol concentrations. Anesthesiology. 2004 Apr;100(4):871-8. https:// doi.org/10.1097/00000542200404000-00017 PMID:15087622

22. Takizawa D, Takizawa E, Miyoshi $S$, Kawahara F, Hiraoka $\mathrm{H}$. The increase in total and unbound propofol concentrations during accidental hemorrhagic shock in patients undergoing liver transplantation. Anesth Analg. 2006 Nov; 103(5):1339-40. https://doi.org/10.1213/01. ane.0000242326.01745.13 PMID:17056994

23. Hiraoka H, Yamamoto K, Okano N, Morita T, Goto F, Horiuchi R. Changes in drug plasma concentrations of an extensively bound and highly extracted drug, propofol, in response to altered plasma binding. Clin Pharmacol Ther. 2004 Apr;75(4):324-30. https://doi. org/10.1016/j.clpt.2003.12.004 PMID:15060510

24. Carranza Corté JL. MSMM. Perfiles Farmacocinéticos de la Ketamina: ¿Existe Respuesta Hemodinámica en el Paciente con Choque Hemorrágico? Rev Anest Mex. 1998;10:191-5.

25. Gales A, Maxwell S. Ketamina: Evidencia y Usos corrientes. Disponible en: www.wfsahq.org

26. Sepúlveda P, Cortinez LI, Irani $M$, et al. Differential Frontal Alpha Oscillations Could Reflect Distinct Mechanisms of Loss of Consciousness: Evidence from Slow- and Fast-rate Propofol infusion. Anaesthesia. 2020 Feb;75(2):196-201. https:// doi.org/10.1111/anae.14885 PMID:31788791 